\title{
The Potential Role of Inhaled Nitric Oxide in Managing COVID-19 Associated Lung Complications Review of Literature
}

\author{
Dunia Alhareth, $M D^{1}$ and Sanaa Badour, MD, MSc ${ }^{2 *}$ \\ ${ }^{1}$ Alhareth Private Practice, Jeddah, Saudi Arabia \\ ${ }^{2}$ Department of Internal Medicine, American University of Beirut, Lebanon
}

*Corresponding author: Sanaa Badour, MD, MSc, Department of Internal Medicine, American University of Beirut, Lebanon

\begin{abstract}
The outbreak of novel coronavirus disease 2019 (COVID-19) in the city of Wuhan, Hubei Province, China, was declared a pandemic by the World Health Organization (WHO) on March 21, 2020. As of June 16, 2020, the World Health Organization $(\mathrm{WHO})$ has reported near eight million cases and half a million deaths worldwide. The majority of cases have spontaneously resolved. However, some have developed various fatal complications including organ failure, septic shock, pulmonary edema, severe pneumonia, and Acute Respiratory Distress Syndrome (ARDS).

Given the lack of effective antiviral therapy against COVID-19, current treatments mainly focus on symptomatic and respiratory support. In this review, the role of Nitric Oxide (NO) in COVID-19 management will be explored. We have conducted a thorough literature review basically by searching Medline, PubMed and Scopus for studies on COVID-19 and NO.

Based on the historical use of inhaled NO in the treatment of pulmonary hypertension, ARDS, and other respiratory diseases, several undergoing randomized clinical trials are investigating the role of NO in COVID-19. Given its relatively low cost, readerly availability and acceptable safety profile, nitric oxide may be a game changer in COVID-19 management. We have found both clinical and preclinical evidence to support the beneficial role of $\mathrm{NO}$, possibly derived from its viricidal effect, on the coronavirus-mediated acute respiratory syndrome and the associated microthrombotic phenomenon. Accordingly, we advocate for its use in the early stages of the disease, when clinical worsening is most likely, aiming to prevent the progression of COVID-19 course.
\end{abstract}

The outbreak of novel Coronavirus Disease 2019 (COVID-19) in the city of Wuhan, Hubei Province, China, was declared a pandemic by the World Health Organi- zation (WHO) on March 21, 2020. This has marked the introduction of the third highly pathogenic and largescale epidemic coronavirus into the human population in the twenty-first century after the Severe Acute Respiratory Syndrome Coronavirus (SARS-CoV) in 2002 and Middle East Respiratory Syndrome Coronavirus (MERSCoV) in 2012. As of July 2020, the World Health Organization (WHO) has reported more than ten million cases and half a million deaths worldwide [1].

To date, most SARS-CoV-2 infected patients have developed mild symptoms such as dry cough, sore throat, and fever. The majority of cases have spontaneously resolved [2]. However, some have developed various fatal complications including organ failure, septic shock, pulmonary edema, severe pneumonia, and Acute Respiratory Distress Syndrome (ARDS) [3]. In some reports, ARDS has been described in approximately $40 \%$ patients with 2019-nCoV pneumonia [4], and it was crucial in increasing the risk of death. Notably, patients who required intensive care support were older and had multiple comorbidities including cardiovascular, cerebrovascular, endocrine, digestive, and respiratory disease [5].

Given the lack of effective antiviral therapy against COVID-19, current treatments mainly focus on symptomatic and respiratory support [6]. In this review, the role of Nitric Oxide (NO) in COVID-19 management will be explored. We have conducted a thorough literature review basically by searching Medline, PubMed and Scopus for studies on COVID-19 and NO.

Nitric oxide is an important signalling molecule be-

Citation: Alhareth D, Badour S (2020) The Potential Role of Inhaled Nitric Oxide in Managing COVID-19 Associated Lung Complications Review of Literature. Int J Respir Pulm Med 7:145. doi. org/10.23937/2378-3516/1410145

Accepted: October 07, 2020; Published: October 09, 2020

Copyright: (C) 2020 Alhareth D, et al. This is an open-access article distributed under the terms of the Creative Commons Attribution License, which permits unrestricted use, distribution, and reproduction in any medium, provided the original author and source are credited 
tween cells and is involved in a wide range of processes $[7,8]$. Inhalation of NO leads to selective dilatation of pulmonary vessels in ventilated lung parenchyma and may thus improve arterial oxygenation and blunt pulmonary hypertension [9]. In addition, antimicrobial activity of NO has been described for several bacteria, protozoa and for some viruses $[8,10,11]$.

Up to date, there are no studies that describe the use of pulmonary vasodilators, including Nitric Oxide, in COVID-19 patients [12]. In studying the role of NO in Severe Acute Respiratory Syndrome Coronavirus (SARS $\mathrm{CoV}$ ) in vitro, Akerstrom, et al. demonstrated that NO specifically inhibited the replication cycle of SARS Co, most probably during the early steps of infection, suggesting an antiviral effect of NO [13]. A pilot study in 2004, during the SARS CoV outbreak, showed that lowdose inhaled NO could shorten the time of ventilatory support for patients infected with SARS-CoV [14]. In the mentioned study, inhaled NO improved arterial oxygenation and enabled the reduction of inspired oxygen therapy [14]. In addition, chest radiography showed decreased lung infiltrates, and the physiological effects remained even after termination of inhaled NO therapy, suggesting, not only a pulmonary vasodilator effect of inhaled NO, but also an effect on SARS [14]. A Cochrane review [15] identified 13 RCTs (1243 patients) on inhaled nitric oxide in ARDS; this treatment showed no significant effect on mortality and an increased risk of kidney injury, but resulted in a transient improvement in oxygenation. No study, however, has assessed the use of inhaled nitric oxide as a "rescue" therapy [15]. Given the possible harm from inhaled nitric oxide and the absence of a clear mortality benefit, recently published guidelines [12] on the management of critically ill adults with Coronavirus Disease 2019 issued a strong recommendation against the routine use of nitric oxide in patients with ARDS. However, the guidelines recommend a trial of inhaled nitric oxide as a "rescue" therapy, after trying other options, in view of the finding of improved oxygenation [12]. The guidelines further caution that if inhaled nitric oxide is used without a good response in terms of oxygenation, it should be tapered off to avoid rebound pulmonary vasoconstriction that can occur with prolonged use and abrupt discontinuation [12].

However, even though it can meet the ARDS Berlin definition [16], Gattinoni and colleagues [17] suggest that the COVID-19 pneumonia is a specific disease with peculiar phenotypes. Mainly, it is characterized by the dissociation between the severity of the hypoxemia and the maintenance of relatively good respiratory mechanics. Accordingly, the authors propose the presence of two types of patients (non-ARDS, type 1, and ARDS, type 2) with different pathophysiology [17]. Consequently, the oxygenation response to $\mathrm{NO}$ is variable. The COVID-19 pneumonia appears to interfere with the vascular regulation up to complete loss of vascular tone.
While evidence is still lacking, Gattinoni, et al. suggest that Nitric oxide should not work in fully vasoplegic patients (non-ARDS, type 1) but could possibly work in patients in which pulmonary hypertension is more likely (ARDS, type 2) [17].

In a recently published consensus report by Ryan, et al. [18] on the care of patients with Pulmonary Arterial Hypertension (PAH) during the COVID-19 pandemic, the authors suggest that in patients with shock, related or unrelated to COVID-19, PAH-specific therapies should be continued during hospitalization and patients unable to tolerate oral or inhaled medications may need to be transitioned from oral to intravenous medications to get through severity of a COVID-19 infection [19]. The authors state that it is important to consider Nitric Oxide (NO) during clinical decompensation in lieu of agents that have the potential to lower blood pressure [18].

On a different note, the incidence of cardiovascular symptoms in patients with COVID-19 was reported to be high, owing to the systemic inflammatory response and immune system disorders during disease progression [20]. The proposed underlying mechanisms include ACE2-related signalling pathways and the cytokine storm triggered by an imbalanced response by type 1 and type $2 \mathrm{~T}$ helper cells $[2,21]$. It was further postulated that pulmonary thrombosis may further complicate the course of 2019-nCoV pneumonia via a prothrombotic endothelial dysfunction [22]. This may cause activation of cytokine-producing monocytes, such as interleukin 6 and tumor necrosis factor, which in turn induce activation of the endothelial cells and tissue factors that triggers the blood coagulation cascade [23]. While the activation of blood coagulation is essential in counteracting viral infections along with the immune system trapping viruses by forming a fibrin network [24], a massive inflammatory and coagulative response is dangerous because it can lead to a local thrombosis in the lungs [25]. Furthermore, this severe acute inflammation induces a local consumption coagulopathy, i.e. Disseminated Intravascular Coagulopathy (DIC), resulting in ARDS [22]. ARDS may be associated with pulmonary vascular microthrombosis [26].

It has been suggested that preemptive enhancement of whole-body NO tone may specifically maintain tonic inhibitory NO tone as a novel approach to lessen deleterious physiological conditions associated with viral infection, including SARS-COV-2, and rampant proinflammatory processes [27].

Horn, et al. questioned whether pulmonary arterial hypertension patients are at lower risk from severe COVID-19 infection [28]. They analyzed a very small sample size of 13 COVID-19 and PAH preliminary cases reported (acquired from the Pulmonary Hypertension Clinicians and Researchers Network to Date), and found generally tolerable symptoms, relatively early recovery and only one reported death. The authors suggested 
that the paradoxically pre-existing pulmonary vasculopathy and/or PAH-specific medications somehow may be protective for these otherwise high-risk patients [28]. The authors concluded that it is possible that these PAH-specific medications, including nitric oxide, may offer a protective benefit in COVID-19 patients through mediation of pulmonary vasodilatation, anti-proliferation and antithrombotic effect. Fernandes, et al. however, claim that these assumptions were based on little data at an early stage of the pandemic in the US, and the resulting speculations could mislead and put $\mathrm{PAH}$ patients at higher risk [29].

In discussing some of the pearls that have been provided by health professionals in Italy, the UK, and US, and that are based on anecdotal experience, Marcos, et al. [30] reported that many centers use inhaled nitric oxide and prostacyclin with good effect. However, tachyphylaxis with nitric oxide is usually seen after 4-5 days [30].

Finally, to complete this review, it is worth mentioning an interesting case report by Zamanian and colleagues [31], of a patient with Pulmonary Arterial Hypertension (PAH) who was treated remotely in an ambulatory setting with inhaled Nitric Oxide (iNO). This patient with well-controlled vasoreactive $\mathrm{PAH}$ lived in a remote area. After being diagnosed with COVID-19, she experienced symptoms of worsening breathlessness. In light of her prior confirmed responsiveness to iNO, and considering her concerns about travelling the long distance to establish care, the care givers established a plan to support her with an ambulatory iNO system while monitoring her symptoms, vital signs, and functional capacity remotely. The patient had rapid and sustained improvement in her 6-minute walk distance, as assessed by her caregiver, and symptom score, and she recovered over several days without having to engage emergency department or hospital care. Alvarez, et al. reflected on this case reports when discussing home NO therapy for COVID-19 [32].

Based on the historical use of inhaled NO in the treatment of pulmonary hypertension, ARDS, and other respiratory diseases, several undergoing randomized clinical trials are investigating the role of NO in COVID-19 [33,34]. Given its relatively low cost, readerly availability and acceptable safety profile, nitric oxide may be a game changer in COVID-19 management. In conclusion, both clinical and preclinical evidence, support the beneficial role of NO, possibly derived from its viricidal effect, on the coronavirus-mediated acute respiratory syndrome and the associated microthrombotic phenomenon. Accordingly, we advocate for its use in the early stages of the disease, when clinical worsening is most likely, aiming to prevent the progression of COVID-19 course.

\section{Author Contributions}

Dr. Alharith has come up with the original idea of this review, and participated in writing the manuscript and revising the final version. Dr. Badour has done the literature search and participated in writing the manuscript.

\section{Role of the Funding Source}

This work was a personal initiative by Dr. Alharith and Dr. Badour, where no funding was needed. For the purpose of literature search, Dr. Badour used her credentials as a student and staff member at the American University of Beirut to obtain access to main search engines and medical journals.

\section{Conflict of Interest}

Dr. Alharith and Dr. Badour report no conflict of interest.

\section{References}

1. Coronavirus disease (COVID-19) weekly epidemiological update and weekly operational update.

2. Huang C, Wang Y, Li X, Ren L, Zhao J, et al. (2020) Clinical features of patients infected with 2019 novel coronavirus in Wuhan, China. The Lancet 395: 497-506.

3. Chen N, Zhou M, Dong X, Qu J, Gong F, et al. (2020) Epidemiological and clinical characteristics of 99 cases of 2019 novel coronavirus pneumonia in Wuhan, China: A descriptive study. The Lancet 395: 507-513.

4. Wu C, Chen X, Cai Y, Zhou X, Xu S, et al. (2020) Risk factors associated with acute respiratory distress syndrome and death in patients with coronavirus disease 2019 pneumonia in Wuhan, China. JAMA Intern Med 180: 934-943.

5. Sohrabi C, Alsafi Z, O'Neill N, Khan M, Kerwan A, et al. (2020) World Health Organization declares global emergency: A review of the 2019 novel coronavirus (COVID-19). Int J Surg 76: 71-76.

6. Guo YR, Cao QD, Hong ZS, Tan YY, Chen SD, et al. (2020) The origin, transmission and clinical therapies on coronavirus disease 2019 (COVID-19) outbreak-an update on the status. Mil Med Res 7: 1-10.

7. Boucher J, Moali C, Tenu J (1999) Nitric oxide biosynthesis, nitric oxide synthase inhibitors and arginase competition for L-arginine utilization. Cell Mol Life Sci 55: 1015-1028.

8. Pope M, Marsden P, Cole E, Sloan S, Fung L, et al. (1998) Resistance to murine hepatitis virus strain 3 is dependent on production of nitric oxide. J Virol 72: 7084-7090.

9. Rossaint R, Falke KJ, Lopez F, Slama K, Pison U, et al. (1993) Inhaled nitric oxide for the adult respiratory distress syndrome. N Engl J Med 328: 399-405.

10. Lane TE, Paoletti AD, Buchmeier MJ (1997) Disassociation between the in vitro and in vivo effects of nitric oxide on a neurotropic murine coronavirus. J Virol 71: 2202-2210.

11. Adler H, Beland JL, Del-Pan NC, Kobzik L, Brewer JP, et al. (1997) Suppression of herpes simplex virus type 1 (HSV-1)induced pneumonia in mice by inhibition of inducible nitric oxide synthase (iNOS, NOS2). J Exp Med 185: 1533-1540.

12. Alhazzani W, Møller MH, Arabi YM, Loeb M, Gong MN, et al. (2020) Surviving sepsis campaign: Guidelines on the management of critically ill adults with Coronavirus Disease 2019 (COVID-19). Intensive Care Med 46: 854-887.

13. Åkerström S, Mousavi-Jazi M, Klingström J, Leijon M, Lundkvist $\AA$, et al. (2005) Nitric oxide inhibits the replication 
cycle of severe acute respiratory syndrome coronavirus. J Virol 79: 1966-1969.

14. Chen L, Liu P, Gao H, Sun B, Chao D, et al. (2004) Inhalation of nitric oxide in the treatment of severe acute respiratory syndrome: A rescue trial in Beijing. Clin Infect Dis 39: 1531-1535.

15. Gebistorf F, Karam O, Wetterslev J, Afshari A (2016) Inhaled nitric oxide for acute respiratory distress syndrome (ARDS) in children and adults. Cochrane Database Syst Rev.

16. Force ADT, Ranieri V, Rubenfeld G, Thompson B, Ferguson N, et al. (2020) Acute respiratory distress syndrome. JAMA 307: 2526-2533.

17. Gattinoni L, Chiumello D, Rossi S (2020) COVID-19 pneumonia: ARDS or not? Crit Care 24: 154.

18. Ryan JJ, Melendres-Groves L, Zamanian RT, Oudiz RJ, Chakinala M, et al. (2020) Care of patients with pulmonary arterial hypertension during the coronavirus (COVID-19) pandemic. Pulm Circ 10.

19. Pan IZ, Carey JR, Jacobs JA, Dechand J, Sessions JJ, et al. (2020) Transitioning between prostanoid therapies in pulmonary arterial hypertension. Front Med 7: 81.

20. Zheng YY, Ma YT, Zhang JY, Xie X (2020) COVID-19 and the cardiovascular system. Nat Rev Cardiol 17: 259-260.

21. Wong CK, Lam CWK, Wu AKL, Ip WK, Lee NLS, et al. (2004) Plasma inflammatory cytokines and chemokines in severe acute respiratory syndrome. Clin Exp Immunol 136: 95-103.

22. Marongiu F, Grandone E, Barcellona D (2020) Pulmonary thrombosis in 2019-nCoV pneumonia? J Thromb Haemost.

23. Levi M (2018) Pathogenesis and diagnosis of disseminated intravascular coagulation. Int J Lab Hematol 40: 15-20.

24. Antoniak S (2018) The coagulation system in host defense. RPTH 2: 549-557.
25. Stroo I, Ding C, Novak A, Yang J, Roelofs JJ, et al. (2018) Inhibition of the extrinsic or intrinsic coagulation pathway during pneumonia-derived sepsis. Am J Physiol Lung Cell Mol Physiol 315: 799-809.

26. Chang JC (2019) Acute respiratory distress syndrome as an organ phenotype of vascular microthrombotic disease: Based on hemostatic theory and endothelial molecular pathogenesis. Clin Appl Thromb Hemost 25.

27. Stefano GB, Esch T, Kream RM (2020) Potential immunoregulatory and antiviral/sars-cov-2 activities of nitric oxide. Med Sci Monit 26: e925679-1-e925679-3.

28. Horn E, Chakinala MM, Oudiz R, Joseloff E, Rosenzweig EB (2020) Could pulmonary arterial hypertension (PAH) patients be at a lower risk from severe COVID-19? Pulm Circ 10.

29. Fernandes T, Papamatheakis D, Poch D, Kim NH (2020) Letter to the editor regarding "Could pulmonary arterial hypertension $(\mathrm{PAH})$ patients be at lower risk from severe COVID-19?" Pulm Circ 10.

30. Cascella M, Rajnik M, Cuomo A, Dulebohn SC, Di Napoli $\mathrm{R}$ (2020) Features, evaluation and treatment coronavirus (COVID-19). Statpearls.

31. Zamanian RT, Pollack Jr CV, Gentile MA, Rashid M, Fox JC, et al. (2020) Outpatient inhaled nitric oxide in a patient with vasoreactive IPAH and COVID-19 infection. Am J Respir Crit Care Med 202: 130-132.

32. Alvarez RA, Berra L, Gladwin MT (2020) Home nitric oxide therapy for COVID-19. AJRCM 202.

33. Mallinckrodt evaluates the potential role for inhaled nitric oxide to treat COVID-19 associated lung complications, engages with scientific, governmental and regulatory agencies.

34. Chong L, Binxiao S, Hailong D, Bijan SF, Luigi GG, et al. (2020) Protocol for a randomized controlled trial testing inhaled nitric oxide therapy in spontaneously breathing patients with COVID-19. medRxiv. 